\title{
Genetic Defects of Apoptosis and Primary Immunodeficiency
}

\author{
Helen C. Su, MD, PhD ${ }^{\mathrm{a}}$ and Michael J. Lenardo, $\mathbf{M D}^{\mathrm{b}}$ \\ a Clinical Investigator, Human Immunological Diseases Unit, Laboratory of Host Defense, National Institute \\ of Allergy and Infectious Diseases, National Institutes of Health, Bethesda, MD \\ b Senior Investigator, Molecular Development Section, Laboratory of Immunology, National Institute of \\ Allergy and Infectious Diseases, National Institutes of Health, Bethesda, MD
}

\section{Synopsis}

Programmed cell death is important for maintaining lymphocyte homeostasis. Several human inherited diseases with impaired apoptosis have been identified at the genetic level: autoimmune lymphoproliferative syndrome (ALPS), caspase-8 deficiency state (CEDS), and X-linked lymphoproliferative syndrome (XLP). These diseases feature excess lymphocyte accumulation, autoimmunity, and/or immunodeficiency. Elucidating their molecular pathogenesis has also provided new insights into the signaling mechanisms regulating apoptosis and lymphocyte activation.

\section{Keywords}

apoptosis; immunodeficiency; autoimmune lymphoproliferative syndrome (ALPS); caspase-8 deficiency state (CEDS); X-linked lymphoproliferative disease (XLP)

\section{Background}

Programmed cell death (reviewed in ${ }^{[1]}$ ) is a physiologically important process in which lymphocytes are triggered to undergo suicide by various stimuli, including death receptor ligation, restimulation of antigen receptors (often termed "activation-induced cell death," AICD), or withdrawal of trophic factors such as IL-2. One form of programmed cell death is apoptosis, which is characterized ultrastructurally by cell shrinkage, nuclear condensation and fragmentation, membrane blebbing, and shedding of apoptotic bodies containing relatively intact organelles. ${ }^{[2]}$ Biochemically, apoptosis is marked by activation of caspases, intracellular enzymes that degrade cellular components including structural proteins and cell maintenance enzymes. ${ }^{[2]}$ In vivo, apoptotic cells are rapidly engulfed by phagocytic cells while eliciting minimal inflammatory sequelae. Other forms of programmed cell death include necrosis and autophagy. ${ }^{[2]}$ These alternative forms of death can occur during intracellular infections with pathogens, but their physiologic relevance in humans is generally less well understood.

Although apoptosis takes place in different tissues and during embryonic development, it serves a particularly important role in the immune system. Apoptosis eliminates strongly selfreactive immature T cells during thymic development (central tolerance). Self-reactive T cells that have escaped this negative selection, or autoreactive B cells, can undergo apoptosis in the periphery as mature lymphocytes (peripheral deletional tolerance). Additionally, apoptosis

Corresponding author for proof and reprints: Helen C. Su, MD, PhD, Building 10 CRC, Room 5W-3932, 10 Center Dr., MSC 1456 , Bethesda, MD 20892-1456, (301) 451-8783, (301) (fax) 402-2240, E-mail: hsu@ niaid.nih.gov (email), Coauthor's address: Michael J. Lenardo, MD, Building 10, Room 11N311, 10 Center Dr., MSC 1892, Bethesda, MD 20892-1892, (301) 486-6754, (301) (fax) 480-7352, E-mail: mlenardo@niaid.nih.gov (email). 
serves to limit the magnitude and duration of the immune response to foreign antigens. Mature lymphocytes normally respond to these by first becoming activated and proliferating. Later, their numbers return to baseline, except for a small pool of memory cells that persists. This is achieved through a combination of death receptor-, antigen receptor restimulation-, and cytokine withdrawal- dependent death mechanisms that occur at or after the height of the immune response. Although naive lymphocytes start out relatively resistant to apoptosis, entry into cell cycle sensitizes them to die. Thus, activated, cycling $\mathrm{T}$ and $\mathrm{B}$ cells are governed by a negative feedback control mechanism termed propriocidal regulation. ${ }^{[3]}$ This is crucial for maintaining normal overall lymphocyte homeostasis over an person's life.

The prototypic disorder of impaired apoptosis in humans is autoimmune lymphoproliferative syndrome (ALPS), which has been described extensively in a previous volume. ${ }^{[4]}$ This review will focus on new advances in understanding ALPS, and a related primary immunodeficiency with a genetic defect in apoptosis called caspase-8 deficiency state (CEDS). In addition, we will briefly discuss a variant of ALPS called autoimmune lymphoproliferative disease (ALD), as well as another primary immunodeficiency featuring apoptosis abnormalities, X-linked lymphoproliferative syndrome (XLP). Related disorders with hemophagocytic lymphohistiocytosis, which impair cytotoxic granule formation and the perforin-granzyme death pathway, go beyond the scope of this review. ${ }^{[5]}$ For further details on all, we refer the reader to several other recent reviews. ${ }^{[1,6,7]}$

\section{Autoimmune lymphoproliferative syndrome (ALPS)}

\section{Clinical Features}

Patients typically present in infancy or early childhood (median age of 24 months) with persistent lymph node and/or splenic enlargement. ${ }^{[8]}$ There is often accompanying hepatomegaly without liver disease, and occasional thymic enlargement on CT scan. ${ }^{[9]}$ Patients lack the constitutional symptoms that would suggest infectious or neoplastic etiologies. Most patients have $\mathrm{T}$ and $\mathrm{B}$ cell lymphocytosis, as well as polyclonal

hypergammaglobulinemia. ${ }^{[10-13]}$ Eosinophilia or monocytosis may be noted. ${ }^{[14,15]}$ Up to $80 \%$ of patients have detectable autoantibodies, most commonly anticardiolipin or direct Coombs antibodies. ${ }^{[8,16-18]}$ By contrast, only half of these have actual autoimmune disease, usually autoimmune hemolytic anemia (AIHA), idiopathic thrombocytopenic purpura (ITP), or autoimmune neutropenia (AIN). ${ }^{[8]}$ Glomerulonephritis, optic neuritis or uveitis, GuillainBarré, primary biliary cirrhosis, coagulopathy with factor VIII inhibitor, autoimmune hepatitis, vasculitis, and linear IgA dermopathy have been reported in ALPS patients; however, these autoimmune diseases targeting non-hematopoietic organ systems are uncommon. ${ }^{[8,19,20]} \mathrm{By}$ contrast, the MRL-lpr or gld mouse counterparts of ALPS are often considered animal models of systemic lupus erythematosus (SLE) because of characteristic autoantibodies (anti-dsDNA, anti-Sm, anti-immunoglobulin) and prominent non-hematopoietic autoimmune disease including glomerulonephritis, polyarteritis, and sialoadenitis. ${ }^{[21]}$

\section{Diagnosis}

The differential diagnosis for ALPS is extensive and includes systemic infections (EBV, CMV, tuberculosis, toxoplasmosis, hepatitis C, HIV), medications, benign lymphoproliferative disorders such as sinus histiocytosis with massive lymphadenopathy (SHML, also known as Rosai-Dorfman disease) ${ }^{[22]}$ or progressive transformation of germinal centers (PTGC), leukemia or lymphoma, hematologic disorders such as Evans syndrome ${ }^{[23]}$ or hereditary spherocytosis, and autoimmune diseases such as SLE or rheumatoid arthritis (Felty syndrome). ${ }^{[24,25]}$ Several diagnostic criteria have been proposed for ALPS: 1) chronic nonmalignant lymphadenopathy or splenomegaly, 2) expansion of $\mathrm{CD}^{-} \mathrm{CD}^{-} \mathrm{TCR} \alpha / \beta$ rearranged cells (DNT), and 3) defective lymphocyte apoptosis. ${ }^{[1,8]}$ Supporting criteria include 
autoimmune manifestations, characteristic histopathologic findings, relevant gene mutations (see below), and family history. Of note, a variant of ALPS called autoimmune lymphoproliferative disease (ALD) lacks DNT expansion but shares lymphoid organ enlargement, autoimmunity, and defective lymphocyte apoptosis. ${ }^{[26,27]}$ The diagnostic criteria for ALPS should be qualified in light of recent findings discussed below:

1) Lymphoaccumulation-Lymphadenopathy in ALPS is typically nontender and generalized, especially involving the neck and axillary regions. At the NIH, this is scored as 2 + if 1 to $2 \mathrm{~cm}$ in diameter, $3+$ if visible without palpation, or $4+$ if distorting normal anatomical landmarks. ${ }^{[11]}$ Splenomegaly is measured in the cranio-caudal dimension, and is also monitored for changes over time. CT scans are also used to assess for intrathoracic or intraabdominal lymphadenopathy in equivocal cases with borderline lymphadenopathy or splenomegaly on physical examination. Lymph nodes and spleen sizes remain relatively stable over years, but may decrease somewhat with age..$^{[9,28,29]}$

2) DNT expansion-Patients with ALPS have lymphocytosis that affects $T$ and $B$ but not NK cells. ${ }^{[11,12]}$ There is expansion of $\mathrm{CD}^{+} \mathrm{T}$ cells that express $\mathrm{CD} 57$, but no net expansion of $\mathrm{CD} 4 \mathrm{~T}$ cells because of low $\mathrm{CD} 4{ }^{+} \mathrm{CD} 25^{+}$numbers. $\mathrm{T}$ cells express upregulated HLA-DR. Both total B cells and $\mathrm{CD}^{+} \mathrm{B}$ cells are increased. A distinctive but not pathognomonic feature of ALPS, which also is seen in the lpr or gld mouse models, is the expansion of an unusual polyclonal population of mature DNT cells that expresses rearranged TCR $\alpha / \beta \cdot{ }^{[12,30]} \mathrm{DNT}$ cells are thought to be senescent $\mathrm{T}$ cells that have down-modulated their CD4 or CD8 co-receptors. They express CD57, CD27, and HLA-DR, as well as the CD45R B220 isoform usually found on $\mathrm{B}$ but not T cells. ${ }^{[31]}$ It is important to distinguish DNT from TCR $\gamma / \delta$ rearranged cells, which are normally $\mathrm{CD}^{-} \mathrm{CD}^{-}$, as the latter can be non-specifically increased in ALPS as well as other conditions such as infection, autoimmunity, and malignancy including $\mathrm{T}$ cell large granular lymphocyte leukemia (LGL). At the NIH Clinical Center Laboratory, DNT cells constitute less than $1 \%$ of peripheral lymphocytes, or less than $18 \mathrm{cells} / \mu \mathrm{l}^{3}$ in healthy adults. By contrast, they can reach over $40 \%$ of lymphocytes in ALPS patients, ${ }^{[32]}$ although most patients exhibit a more modest DNT elevation that may not be consistently elevated between blood draws. We and others have observed mildly elevated DNT in children who do not fulfill other criteria of ALPS, or who have other primary immunodeficiencies such as DiGeorge syndrome (Jack Bleesing, MD, PhD, Cincinnati, OH, personal communication, October 2007). Thus establishing age-dependent normal ranges for DNT in diagnostic flow cytometry laboratories will aid in interpreting borderline DNT. Given this confusion, it is not clear whether patients with autoimmune lymphoproliferative disease (ALD), who reportedly lack DNT expansion but who otherwise fulfill criteria for ALPS, actually have a forme fruste of ALPS. ${ }^{[26,27]}$ Supporting this possibility, mice with conditional Fas deletions in B cells or dendritic cells lacked DNT expansion but developed other markers of ALPS, i.e., lymphoaccumulation, hypergammaglobulinemia, and autoimmunity. ${ }^{[33,34]}$ Alternatively, ALD may represent a related but separate entity having a genetic defect distinct from ALPS.

3) Defective lymphocyte apoptosis-Because the clinical features of this disease stem from defective apoptosis, testing for this functional abnormality remains a sina qua non for diagnosing ALPS. T cells are activated and cycled in IL-2 to render them susceptible to apoptosis. Alternatively, herpesvirus saimiri (HVS)-or Epstein-Barr virus (EBV)immortalized T or B cell lines can be tested, although any defects should be confirmed in primary lymphocytes. ${ }^{[13,35]}$ Although initial studies demonstrating an apoptotic defect tested death in response to $\mathrm{T}$ cell receptor (TCR) restimulation, results are more reproducible when cells are stimulated directly through the Fas death receptor (also known as CD95/ APO1). ${ }^{[28,36]}$ Induction of cell death is tested after stimulating overnight with cross-linked APO1.3 antibodies. ${ }^{[15]}$ Numbers of living cells excluding the dye propidium iodide are counted 
using a flow cytometer. Treated samples are compared to untreated samples to calculate the \% cell loss. A greater than $50 \%$ difference in \% cell loss of patient compared to normal healthy controls indicates an apoptosis defect. ${ }^{[15]}$ Lesser but reproducible differences may still be indicative of a milder apoptosis defect.

The standard killing assay using anti-Fas agonistic antibodies to trigger Fas signaling will reveal defects in most patients with ALPS. This includes disease due to germline Fas mutations or caspase-10 mutations, which together account for up to $\sim 65 \%$ of ALPS cases. To reveal apoptotic defects due to Fas ligand (FasL) mutations, killing of a Fas-expressing cell line by $\mathrm{T}$ cell blasts from the patient (which have been activated to express FasL) can be tested. ${ }^{[37]}$ Alternatively, killing of the Fas-expressing cell line by cells transfected with mutant FasL constructs is measured. ${ }^{[38,39]}$ In cases where our first line apoptosis screening is normal, we assess IL-2-withdrawal-induced death in primary T cells after washing cells extensively and resuspending in fresh media supplemented without as compared to with IL-2. ${ }^{[40]}$ Whereas death can be seen after a day of anti-Fas stimulation of normal T cells, IL-2-withdrawal-induced death takes about 3 days to become evident. Research testing is available to assess pathways of apoptosis induced by other stimuli such as $\gamma$-irradiation or certain drugs.

A diagnostic limitation of apoptosis testing is that it can only be reliably assessed in activated, cycling T cells. $2 \%$ of patients with ALPS have somatic Fas mutations that are only consistently found in the DNT cells. ${ }^{[41,42]}$ These cases are problematical, because DNT cannot be maintained in culture. Despite their expansion in vivo, DNT cells do not respond to stimuli that normally activate or cause proliferation of conventional T cells. ${ }^{[10,41,43,44]}$ Thus, upon initial TCR stimulation, conventional T cells, but not DNT, expand to take over the culture. ${ }^{[41]}$ Most of these are not mutated and have no apoptotic defect, accounting for a readout of apparent normal apoptosis. In all cases, apoptosis can be properly evaluated only if cells are proliferating well. Lymphocytes that do not cycle well may not have received appropriate signals needed to render them susceptible to propriocidal death. ${ }^{[3]}$ Therefore, in the setting of immunodeficiency, apparent apoptotic defects should be interpreted with caution.

Finally, apoptotic defects can also be measured in dendritic cells isolated from ALPS patients. ${ }^{[32]}$ Dendritic cells function as potent antigen presenting cells (APC) for T cells, and recent studies in mice indicate that impaired dendritic cell apoptosis contributes to increased lymphoproliferative and autoimmune responses. ${ }^{[34,45]}$ This suggests a similar contribution to disease pathogenesis in ALPS patients.

4) Supporting diagnostic tests-Both histopathology and cytokine profile are distinct for ALPS, but these features generally correlate with the extent of peripheral blood DNT expansion. ${ }^{[46]}$ Concern for lymphoma oftens prompts biopsy of enlarged lymph nodes. Histopathology reveals a characteristic paracortical expansion marked by increased DNT cells, accompanied by follicular hyperplasia and plasmacytosis. ${ }^{[46]}$ These early lymphocyte expansions are non-malignant, and differ from the lymphomas that can develop later in some ALPS patients. DNT infiltration can also be seen in both splenic and liver histopathology. ${ }^{[46]}$ In some cases, lymph node histology can also display parafollicular histiocyte proliferation with emperipolesis suggestive of SHML. ${ }^{[22]}$ ALPS patients also have increased serum IL-10, which is largely due to increased production by the expanded DNT, as well as a skewed T helper 2 (Th2) type cytokine profile. ${ }^{[4-49]}$

Elevated serum cobalamin (vitamin B12) can be used as an additional screening test for ALPS. ${ }^{[1,50]}$ Although also increased with liver disease, myeloproliferative disorders, hypereosinophilic syndrome, and metastatic cancers, accompanying signs and symptoms help to differentiate these from ALPS. 
5) Genetic testing-Given the technical demands of lymphocyte apoptosis assays, as well as the limited availability of this testing outside of specialized laboratories such as at the NIH, gene sequencing has been increasingly used to diagnose ALPS as well as other diseases.

Genetic analysis requires understanding that the human genome normally exhibits considerable sequence variability. Variants existing at a population frequency of $1 \%$ or less are called mutations, and those at $>1 \%$, polymorphisms. The discovery of a mutation in a patient implies but does not necessarily mean that it has anything to do with disease. Furthermore, some polymorphisms can be functional, meaning that they can influence disease. Molecular studies must demonstrate that any given DNA sequence alteration significantly changes the coding sequence or its level of expression, and that these changes deleteriously affect cellular function for disease phenotype. Thus, when using genetic analysis as a stand-in for apoptosis assays, it is important to determine whether any given sequence alteration is meaningful. This requires reviewing previously published literature or databases for evidence establishing its pathogenicity.

For ALPS, more sophisticated analyses may also be required to reveal somatic mutations when the mutated cells make up $<20 \%$ of the peripheral blood mononuclear cell (PBMC) population. ${ }^{[41]}$ This entails physically isolating DNT cells by fluorescence or magnetic cell sorting technologies, before preparing genomic DNA for sequencing. Diagnostic algorithms have been proposed to incorporate this step where clinical suspicion for ALPS remains high despite normal apoptosis testing (see above). ${ }^{[42,51]}$

\section{Prognosis and Treatment}

In ALPS, autoimmune and lymphoproliferative disease can follow a variable course but often improve over a span of decades. ${ }^{[9,28]}$ For severe autoimmune disease, a corticosteroid pulse (methylprednisolone 5-30 mg/kg intravenously) can help rapidly control autoimmune disease. This is followed by a daily course of $1-2 \mathrm{mg} / \mathrm{kg}$ oral prednisone for up to several months. ${ }^{[15]}$ Patients may also benefit from simultaneous treatment with intravenous immunoglobulin (IVIG) (1-2 g/kg) for hemolytic anemia or thrombocytopenia, or G-CSF $(1-2 \mu \mathrm{g} / \mathrm{kg}$ subcutaneously once daily or three times a week) for neutropenia. ${ }^{[15]}$ Patients whose autoimmune disease recurs after tapering and stopping corticosteroids may need to be kept on low-doses every other day. For long-term maintenance, mycophenolate mofetil ( $\sim 60 \mathrm{mg} /$ $\mathrm{M}^{2} /$ dose orally, twice daily) can be used to avoid side effects of corticosteroids or splenectomy. ${ }^{[52,53]}$ Failing this, cytotoxic or biologic agents such as cyclophosphamide, azathioprine, vincristine, anti-thymocyte globulin, or rituximab $\left(375 \mathrm{mg} / \mathrm{M}^{2} /\right.$ week $\left.\times 4\right)$ have been tried on a case-by-case basis. ${ }^{[15,54,55]}$ Rapamycin can reduce autoimmune disease, lymphadenopathy, and splenomegaly in $l p r$ mice, but has not yet been tested in ALPS patients. ${ }^{[56]}$ Allogeneic bone marrow transplantation has been used as a last resort for severe intractable disease due to homozygous Fas mutations, but in cases of matched unrelated donor transplant, is itself associated with considerable morbidity and mortality. ${ }^{[57-59]}$

Lymphoproliferative disease can be disfiguring, but by itself is usually not treated because it tends to recur after discontinuing immunosuppressants including corticosteroids, mycophenolate mofetil, cyclosporine A, or azathioprine. ${ }^{[15]}$ Despite initial promising case reports, pyrimethamine, with or without sulfadoxine, has no efficacy in shrinking lymph nodes or spleen or in controlling autoimmune disease in either ALPS patients or MRL-lpr mice. ${ }^{[60]}$ Hypersplenism, although increasing risk for splenic rupture, is generally not treated. When it contributes to chronic refractory severe cytopenias, splenectomy may be considered, but increases risks for post-splenectomy-associated pneumococcal sepsis and death. ${ }^{[15]}$ Patients should receive pre-splenectomy immunizations to Haemophilus influenzae, Neissseria meningitidis, and Streptococcus pneumoniae, and titers used to guide post-splenectomy reimmunizations. ${ }^{[15]}$ Splenectomized patients need to be maintained on lifelong amoxicillin or 
fluoroquinolones for pneumococcal prophylaxis, and treated for potential bacteremia for significant fevers. ${ }^{[15]}$

Although lymphoproliferation is initially benign, approximately $10 \%$ of type 1 A ALPS patients develop lymphoma. ${ }^{[8]}$ Patients are at 51-fold increased risk for developing Hodgkin's lymphoma (HL) at a median age of 11 years old, or 14-fold increased risk for developing nonHodgkin's lymphoma (NHL) at a median age of 21, with an average age of 5 for ALPS diagnosis and 28 for lymphoma diagnosis. ${ }^{[61]}$ The increased predisposition may result from differential signaling thresholds for heterozygous Fas mutations, with continued Fasdependent signaling to promote growth despite impaired apoptosis induction. ${ }^{[62]}$ Lymphomas are primarily of B cell origin, but can also be of $\mathrm{T}$ cell origin, and display diverse histologic types. ${ }^{[61]}$ Most cases of ALPS-asssociated lymphoma have developed in type 1A patients bearing intracellular death domain mutations in Fas; no patients with FasL or caspase-10 mutations have yet developed lymphoma. Therefore, lymphomas are characteristic of type 1A, but not necessarily of types IB, II, or III, although the numbers of patients are too small for a definitive assessment. By contrast, the ALPS patient with NRAS mutation (ALPS type IV) had childhood leukemia and developed NHL at age 32 years. ${ }^{[40]}$ Because of the propensity for developing lymphoma, lymph nodes should be carefully monitored over time, which can be accomplished effectively by periodic CT scans or ultrasound. ${ }^{[9]}$ Suspicious lymph nodes should be biopsied for clonality and chromosomal studies. PET scanning to assess lymphomaassociated increased glucose uptake can also aid in distinguishing lymphoma from the benign lymphadenopathy in ALPS. ${ }^{[63]}$ ALPS patients do respond to conventional treatment for lymphomas, probably because the lymphomas exhibit no loss of heterozygosity and remain sensitive to other apoptosis inducers, although they retain resistance to Fas-induced

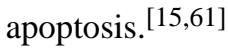

Finally, in vitro studies suggest that RAS antagonists such as farnesyltransferase inhibitors might treating ALPS type IV. [40] These experimental agents are currently being tested against cancers that bear somatic mutations in KRAS and NRAS. Studies testing these agents in ALPS will become feasible as additional patients are identified.

\section{Genetics and Molecular Mechanisms}

ALPS can be classified based upon the underlying genetic mutation (see Table 1). ${ }^{[1]}$ Most mutations affect components of the death-inducing signaling complex (DISC), and understanding mechanistically how the DISC works is key to understanding how these mutations exert their effects (see Figure 1). ${ }^{[6]}$ In brief, FasL and Fas death receptors each exist as preformed homotrimers. When FasL engages Fas, Fas recruits the adapter molecule FADD, which in turn recruits initiator procaspases- 8 or -10 . Assembly of these components into the DISC occurs through homotypic interactions of death domains (for Fas and FADD) or death effector domains (for FADD and procaspases-8/-10). The fully assembled DISC oligomerizes procaspases-8/-10, a step required for caspase-8/-10 activation. They then cleave each other, converting themselves into a highly stable, soluble form that can reach effector caspases. When effector caspases are cleaved and activated, they in turn cleave downstream substrates to cause cell death. Thus, the DISC temporally-spatially converts extracellular signals received through death receptors into an intracellular proteolytic cascade that degrades essential cellular components.

ALPS type IA results from mutations in the Fas death receptor. ${ }^{[13,36,64]}$ Over half of these mutations occur in exon 9, within the death domain that is important for FADD binding (see ALPSbase at http://research.nhgri.nih.gov/ALPS/). Affected individuals possess heterozygous mutations, which exert a dominantly interfering effect on apoptosis induction. ${ }^{[65]}$ For this to occur, an extracellular region of Fas called the preligand assembly domain (PLAD), which directs Fas self-association, must be intact. ${ }^{[66]}$ The defective protein poisons the receptor, 
which has three Fas chains, so that the presence of even one bad chain compromises further DISC assembly. Defective recruitment of DISC components leads to impaired oligomerization, which prevents activation of initiator caspases and interrupts signal propagation. ${ }^{[65]}$ In some cases, Fas mutations outside the death domain may not affect DISC assembly, but may hinder effective formation of higher order signaling protein oligomerization transduction structures (SPOTS) further downstream. ${ }^{[67]}$ These structures contribute to optimal caspase activation, probably by increasing their local concentration for activation and cleavage. In a minority of cases, heterozygous compound loss-of-function Fas mutations may exert their effects through haploinsufficiency. ${ }^{[68,69]}$ In general, mutations in the Fas death domain are more severe in terms of functional apoptotic defects and their clinical consequences. ${ }^{[70]}$ Additionally, homozygous Fas mutations with Fas deficiency, sometimes termed ALPS type 0, are associated with more severe disease. ${ }^{[71,72]}$

A variant of ALPS type 1, termed type $\mathrm{I}_{\mathrm{m}}$ (for mosaic), has heterozygous somatic Fas mutations only in the DNT population of blood cells. ${ }^{[41]}{ }^{[42]}$ In any individual patient, the mutation apparently arises in a committed hematopoietic stem cell such that the DNTs and perhaps a proportion of other hematopoietically-derived cells may also carry the mutation, but the DNT are the only population in which all cells are mutated. In type $\mathrm{I}_{\mathrm{m}}$ ALPS patients, lymphocytosis may be limited to DNT, and certain flow cytometric features such as increased HLA-DR expression on T cells may be absent. ${ }^{[41,42]}$ However, patients with type $\mathrm{I}_{\mathrm{m}}$ ALPS display salient features of lymphadenopathy and splenomegaly, characteristic histopathology, DNT expansion, increased IL-10 production, hypergammaglobulinemia, and gradual improvement of disease with age. Some patients also have autoimmune cytopenias. Together, these suggest a key role for DNT in disease pathogenesis.

ALPS type IB patients have mutations in Fas ligand (FasL). The first individual identified with a FasL mutation (84 base pair deletion in Exon 4) had SLE, with lymphadenopathy, splenomegaly, defective TCR restimulation-induced apoptosis, but no DNT expansion. ${ }^{[37]}$ Two subsequent individuals fulfilling diagnostic criteria for ALPS have homozygous (A247E) or heterozygous FasL (R156G) mutations, which affect the extracellular portion thought to participate in FasL trimerization or Fas binding, respectively. ${ }^{[38,39]}$ The latter patient also has unexplained common variable immunodeficiency (CVID), with hypogammaglobulinemia and granulomatous infiltration of lymph nodes, spleen, and liver.

ALPS type II patients have mutations in caspase-10. ${ }^{[1]}$ Two ALPS patients have heterozygous I406L mutations, ${ }^{[73]}$ and one has a heterozygous L285F mutation, ${ }^{[32]}$ both in the large enzyme subunit. Interestingly, one of the two I406L ALPS patients also fulfills diagnostic criteria for SLE, and the patient with L285F has anti-nuclear autoantibodies (more commonly associated with SLE than ALPS). These caspase-10 mutations dominantly interfere with apoptosis, as demonstrated when introduced by transfection into normal $\mathrm{T}$ cell lines, and also for $\mathrm{L} 285 \mathrm{~F}$ into dendritic cells. ${ }^{[32,73]}$ By contrast, a number of other caspase-10 variants have been identified in ALPS (or ALD) patients whose roles in disease pathogenesis are less clear. ${ }^{[27,32,69,73,74]} \mathrm{An}$ earlier study suggested V410I mutations were pathogenic, based in part upon experiments in epithelial cells demonstrating dominant interference. ${ }^{[32]}$ However, this did not occur using $\mathrm{T}$ cell lines, and reanalysis indicates that $\mathrm{V} 410 \mathrm{I}$ is a polymorphism found at $\sim 5-10 \%$ frequency in the normal population. ${ }^{[73,74]}$ Another variant, Y446C, exhibited decreased apoptotic function when overexpressed in caspase-8/-10 deficient but not wild-type $\mathrm{T}$ cells, suggesting that Y446C may act as a functional polymorphism depending upon the genetic context. ${ }^{[73]} \mathrm{An}$ example of this may be an ALPS patient who has both the Y446C variant and a predicted haploinsufficient Fas mutation. ${ }^{[69]}$ Additionally, an ALPS patient with a known haploinsufficient Fas mutation was recently identified with a caspase-10 P501L mutation. ${ }^{[69]}$ The P501L mutation exhibited decreased caspase-10 activity but no dominant interfering effect when introduced into epithelial cells. ${ }^{[69]}$ The mother carried the P501L but 
not Fas mutation, and her T cells also displayed an apoptotic defect; however, she herself was healthy. Overall, these studies indicate that the caspase-10 I406L and L285F mutations clearly cause ALPS, whereas the V410I and Y446C polymorphisms might contribute to but are not sufficient for disease. Identifying additional patients will help clarify the pathogenicity of the P501L mutation.

ALPS type IV is a new designation that has been proposed for ALPS patients with NRAS mutations. ${ }^{[1,40]}$ Unlike ALPS type I and II, this form of ALPS lacks Fas death receptor-induced apoptosis abnormalities, but instead manifests a specific apoptosis defect triggered by IL-2 deprivation. This defect reflects the utilization of a different upstream pathway for apoptosis induction following cytokine withdrawal (see Figure 1). ${ }^{[1,6]}$ Here, pro- and anti- apoptotic sensors of the BCL2 family, especially the pro-apoptotic member BIM, control mitochondrial stability. The balance of these factors determines whether mitochondria become depolarized, leading to formation of a mitochondria-derived complex called the apoptosome that in turn promotes caspase activation for cell death. To date, one patient has been identified with ALPS type IV, which is caused by a germline heterozygous G13D gain-of-function mutation in NRAS. ${ }^{[40]}$ This mutation decreases intrinsic GTPase activity by keeping bound RAS proteins in an active GTP-bound state. Hyperactivation of the MAPK (RAF/MEK/ERK) pathway suppresses induction of the BIM apoptosis regulatory protein, which in turn impairs apoptosis induced upon cytokine withdrawal. ${ }^{[40]}$ Although this patient clearly fulfilled all three diagnostic criteria for ALPS, he also had several atypical features, including a history of childhood leukemia, lymph node histology showing sinus histiocytosis without prominent DNT expansion, and no increased HLA-DR or CD57 expressing T cells by flow cytometry. ${ }^{[40]}$

ALPS type III refers to patients having defects in apoptosis but lacking mutations in Fas, FasL, caspase-10, caspase-8, or FADD. ${ }^{[1]}$ Approximately 33\% of ALPS patients are classified as type III. ${ }^{[39]}$ ALPS type III likely represents a heterogeneous group of patients with different molecular etiologies. It is not known whether any of these patients have somatic mutations in genes besides Fas. Also, there is a range of patients with ALPS phenotype that have modest or no apoptosis defect, who are not yet understood at the molecular level. These are considered to be "ALPS spectrum."

Although ALPS can be caused by single gene mutations (as discussed above), it is clearly a complex human disease with variable disease penetrance and severity. ${ }^{[28,39]}$ Several factors contribute to this. First, a strong determinant is the mutation and its location. Disease is more likely to occur and be more severe when intracellular (especially death domain) rather than extracellular Fas mutations are present. ${ }^{[29,70]}$ For instance, lymphoma develops primarily in ALPS patients with Fas death domain mutations and a few with intracellular mutations, but none with extracellular mutations. ${ }^{[61]}$ However, even family members bearing identical mutations in death receptor components may display apoptotic defects yet manifest no disease or incomplete disease. ${ }^{[28,75]}$ Moreover, inbred genetic background affects disease expression in the $l p r$ or $g l d$ murine counterparts of ALPS types IA or IB in humans. ${ }^{[21]}$ Together, these observations suggest the contribution of other genetic modifiers.

Potential genetic modifiers include not only those that participate in various death pathways, but also those that contribute to co-existing autoimmune and inflammatory conditions. In ALPS (or ALD), perforin, tumor necrosis factor receptor (TNFR) type I, pyrin, and osteopontin have been implicated as genetic modifiers. Perforin is an essential component in granule mediated cytotoxic death for eliminating virally infected cells, and has been suggested to compensate at least partially for Fas deficiency in T cell death during repeated antigenic stimulation. ${ }^{[76]}$ Thus, alterations in genes involved in cytotoxic granule-mediated, Fas-mediated, TCR-restimulated, or other pathways of apoptosis, may cooperatively influence disease phenotype. Consistent 
with this, certain perforin variants (N252S, A91V) were more highly represented in patients with ALPS or ALD as compared to the general population, although numbers were too small for statistical significance and their effects on perforin function controversial. ${ }^{[77-79]}$ Similarly, several ALPS patients were discovered to also have variants in genes known to be associated with autoimmune or inflammatory conditions. The tumor necrosis factor receptor (TNFR), type I R92Q variant is a polymorphism found at higher frequency in patients with TNFR associated periodic fever syndrome (TRAPS) or rheumatoid arthritis, as compared to the general population; it is therefore considered to be functional, although the mechanism by which it contributes to disease is presently unclear. ${ }^{[80]}$ TNFR1 R92Q has been found in several ALPS patients, including one who also has a homozygous caspase-10 V410I polymorphism. ${ }^{[6]}$ The pyrin E148Q functional polymorphism associated with familial Mediterranean fever (FMF) was also discovered in an ALPS patient with a caspase-10 polymorphism. ${ }^{[6]}$ Certain polymorphisms of osteopontin, a cytokine secreted during chronic inflammatory and autoimmune conditions, were associated at a higher frequency in patients with the ALPS-variant ALD.${ }^{[81]}$ By contrast, other genes such as HLA alleles or CTLA-4 polymorphisms, which are highly associated with certain autoimmune diseases, have shown no relationship to ALPS. ${ }^{[6]}$ However, it is likely that additional genetic modifiers will be found that influence ALPS disease penetrance and severity.

In summary, 60-70\% of ALPS patients have known monogenic causes for ALPS. These include Fas, Fas ligand, caspase-10, and NRAS. However, ALPS is clearly a complex disease whose disease penetrance and severity are likely influenced by many genes that influence different pathways of programmed cell death and contribute to other co-existing autoimmune or inflammatory conditions.

\section{Caspase-8 deficiency state (CEDS)}

\section{Clinical Features}

A brother and a sister with CEDS were identified with a distinct clinical entity that prominently features combined lymphocyte immunodeficiency ${ }^{\left[{ }^{[2]}\right]}$ Unlike ALPS patients, who lack primary immunodeficiency, CEDS patients have recurrent sinopulmonary infections, with mild bronchiectasis in one patient. Both patients also have recurrent mucocutaneous herpesvirus infections with Bell's palsy. They have hypogammaglobulinemia and make poor antibody responses to pneumoccocal polysaccharide antigens. Although they possess normal numbers of lymphocyte subsets, their B cells, T cells, and NK cells do not activate well to stimuli including antigen receptors and various immunoreceptors. ${ }^{[82,83]}$

Counterintuitively, the immunodeficiency of CEDS is accompanied by lymphoaccumulation, autoimmunity, and impaired lymphocyte apoptosis resembling that seen in ALPS. Both patients have lymphadenopathy and mild splenomegaly, which have gradually improved as they have entered early adulthood. They exhibited direct Coombs positive antibodies with a compensated mild hemolytic anemia, as well as anti-thyroid antibodies without thyroid disease. One patient has lupus anticoagulant with prolonged PTT but no history of thrombotic disease. DNT cell numbers have been normal or borderline-high, in a range that is not clearly interpretable at the present time (see discussion above).

CEDS patients have several other clinical features distinct from ALPS. In addition, they lack certain features characteristic of immunodeficiency patients with NEMO or I $\mathrm{KB} \alpha$ mutations, which is relevant because CEDS patients also have NF- $\mathrm{kB}$ activation defects (see below). Both patients have icthyosis. This condition is more typically seen in NEMO patients rather than hypohydrotic ectodermal dysplasia (Maria Turner, MD, Bethesda, MD, personal communication, February 2005). However, neither CEDS patient has ectodermal dysplasia or abnormal teeth. Both patients also have multiple splenic cysts consistent with isolated splenic 
peliosis, which have remained stable over years. This is an extremely rare finding of unknown etiology, whose presence in both CEDS patients suggests a role for caspase- 8 in mesenchymal or vascular development.

Given the limited number of humans with CEDS and the potential modulation of disease spectrum by genetic background, the phenotypes of mice rendered genetically deficient in caspase- 8 can be informative. Although complete deficiency in mice is embryonically lethal, ${ }^{[84]}$ in humans it is compatible with embryonic development, postnatal survival, and successful maternal pregnancy. Conditional deletion of caspase- 8 in T or B cells results in immunodeficiency resembling that in humans. ${ }^{[85,86]}$ The mice also develop an ALPS-like condition with lymphadenopathy, splenomegaly, but without DNT expansion or autoantibodies, and with an unusual lymphocyte infiltration of lungs, liver, and kidneys. ${ }^{[87]}$ These findings indicate that lymphocyte immunodeficiency is a consistent feature of caspase- 8 deficiency, whereas the ALPS-like features of lymphoaccumulation, autoimmunity, and DNT expansion are variable.

\section{Diagnosis}

The differential diagnosis for CEDS includes ALPS, common variable immunodeficiency (CVID), Wiskott-Aldrich syndrome (WAS), and NEMO or IкBa mutations. CVID, especially those due to TACI mutations, are frequently associated with benign lymphoproliferation and autoimmune disease affecting the hematopoietic system. ${ }^{[88]}$ Wiskott-Aldrich syndrome features thrombocytopenia with small platelets, as well as autoimmune disease and variable immune defects. ${ }^{\left[{ }^{[9]}\right.}$ Antibody levels and function can be decreased, as well as T and NK cell function. NEMO or IאBa mutations variably impair B, T, and NK cell function, but can often be distinguished by characteristic skin or dental abnormalities. ${ }^{[90]}$

Clinical features suggesting CEDS should be investigated by immunologic studies assessing serum immunoglobulin levels, antibody function, and lymphocyte activation. Lymphocyte apoptosis testing reveals defects, but must be carefully performed to avoid potentially confounding factors of impaired lymphocyte proliferation. For these reasons, CEDS is more easily diagnosed by caspase- 8 gene sequencing.

\section{Prognosis and Treatment}

The prognosis is uncertain. CEDS patients have generally done well while maintained on IVIG and prophylactic acyclovir to decrease sinopulmonary infections and mucocutaneous herpesvirus outbreaks. Lymphoaccumulation seems to be gradually improving with age. However, mice conditionally deficient in caspase- 8 within T cells developed lethal lymphocytic infiltrative disease of the lungs, liver, and kidneys when they got older. ${ }^{[87]}$ Lymphoma is a potential complication based upon our experience with ALPS patients. However, we have seen no evidence of either in the CEDS patients described.

\section{Genetics and Molecular Pathogenesis}

Disease is inherited in an autosomal recessive manner. ${ }^{[82]}$ The two reported patients, who are siblings, have consanguineous homozygous $\mathrm{C} \rightarrow \mathrm{T}$ transitions that cause $\mathrm{R} 248 \mathrm{~W}$ loss-offunction mutations in caspase- $8 .{ }^{[82]}$ The substitution lies in the large enzyme subunit, which affects an extended loop structure that normally stabilizes substrate when bound. The mutations render the caspase- 8 protein enzymatically inactive and unstable, leading to functional caspase- 8 deficiency. ${ }^{[82]}$ Heterozygous carriers are healthy and lack immune function abnormalities. ${ }^{[82]}$

Caspase- 8 deficiency impairs lymphocyte activation because of failure to induce the gene transcription factor nuclear factor $\mathrm{\kappa B}(\mathrm{NF}-\mathrm{\kappa B})$ when various immunoreceptors are stimulated. 
These include antigen receptors on $\mathrm{T}$ and $\mathrm{B}$ cells, as well as innate immune receptors such as toll-like receptors (TLR) $(-2,-3$, and -4$)$ on B cells or Fc $\gamma$ RIII found on NK cells. ${ }^{[83,86]}$ By contrast, NF- $\mathrm{kB}$ activation is normal in response to the cytokine TNF- $\alpha$ in T cells, or CD40 ligand in B cells. ${ }^{[83]} \mathrm{NF}-\mathrm{\kappa B}$ is typically found in the cytosol, complexed to an inhibitor called inhibitor of $\kappa B(\mathrm{I} \kappa \mathrm{B}) \alpha$. Stimulation through certain immunoreceptors activates the "inhibitor

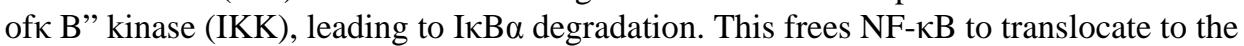
nucleus, where it binds to NF- $\mathrm{KB}$ DNA-binding motifs in promoters to turn on transcription of various genes. In the case of antigen receptors, caspase- 8 functions to link IKK with an upstream signaling complex. This occurs in what is termed the activation-receptor induced signalosome (ARIS) (see Figure 1). ${ }^{[83]}$ The adapter function of caspase-8, as well as its enzymatic activity, are required for optimal IKK activation and downstream NF- $\kappa \mathrm{B}$ activation. The specific defect, elicited by certain immune stimuli, is consistent with the less severe and more immune-restricted clinical phenotype of CEDS patients as compared to NEMO or $\mathrm{I} \kappa \mathrm{B} \alpha$ mutant patients.

Caspase-8 also normally participates in an entirely different signaling complex, the DISC (see Figure 1). ${ }^{[6]}$ As discussed earlier, upon death receptor ligation, oligomerization of caspase- 8 in this complex renders it enzymatically active and capable of cleaving various cellular substrates upon release from the DISC. Thus, caspase-8 deficiency blocks the initiating events necessary to turn on the caspase cascade that is crucial for propagation of signaling for cell death. In this context, it is not surprising that patients with caspase-8 mutation have defective apoptosis and ALPS-like clinical features.

\section{Other immunodeficiencies with apoptosis abnormalities}

Associations with increased basal apoptosis of lymphocytes have been reported for virtually all primary immunodeficiencies. However, there is insufficient characterization of how specific gene mutations responsible for these immunodeficiencies mechanistically affect apoptosis. Some papers also have reported apoptosis defects, but lack careful assessment to exclude confounding factors such as impaired cell cycling that may exist with immunodeficiencies.

Immunodeficiencies characterized by concomitant immune dysregulation with lymphoproliferative features may reflect impaired apoptosis. One such disorder is X-linked lymphoproliferative syndrome (XLP), which features lymphoproliferation including B cell lymphoma, hypogammaglobulinemia, and a fulminant and fatal infectious mononucleosis, often associated with impaired 2B4-dependent NK and CD8 T cell cytotoxicity against EBVinfected cells. ${ }^{[7]}$ Most cases are due to mutations in SH2D1A (SAP), an adapter molecule that interacts with SLAM-family immunoreceptors. Although the precise function of this molecule is still being elucidated, we have recently discovered that, similar to the SAP-deficient mice, SH2D1A is required for TCR restimulation-induced apoptosis in humans. ${ }^{[91,92]}$ A profound decrease in TCR restimulation apoptosis with unabated immune activation may explain the lymphoproliferative burst in XLP. Thus, XLP now joins ALPS and CEDS (impaired death receptor or cytokine-withdrawal apoptosis), as well as familial hemophagocytic lymphohistiocytosis (HLH) and related disorders (with impaired perforin-granzyme mediated killing), in a group of immune homeostasis disorders featuring impaired programmed cell death.

\section{Summary}

Humans with autoimmune lymphoproliferative syndrome (ALPS) have defective lymphocyte apoptosis leading to lymphoid organ enlargement, autoimmune disease, and lymphoid malignancy. These manifestations result from impaired death receptor inducing signaling complex (DISC) formation caused by mutations in Fas, Fas ligand, or caspase-10. Recently, 
an ALPS patient was discovered who has a cytokine withdrawal-induced apoptotic defect due to a gain-of-function mutation in NRAS that blocks BIM induction. Genetic defects in apoptosis also occur in primary immunodeficiencies that share clinical features with ALPS. Patients with caspase- 8 deficiency state (CEDS) due to mutations in the caspase- 8 gene have impaired lymphocyte activation as well as impaired apoptosis. The immunodeficiency is caused by failure to form a caspase-8-dependent (nuclear factor $\kappa \mathrm{B}$ ) NF- $\kappa \mathrm{B}$ gene transcription factor signaling complex, which is distinct from the DISC. Additionally, an SH2D1A (SAP)specific defect in antigen receptor restimulation-induced apoptosis can contribute to disease pathogenesis in X-linked lymphoproliferative syndrome (XLP). These primary immunodeficiencies highlight molecular mechanisms by which signaling for lymphocyte activation and apoptosis are coordinately regulated.

\section{Acknowledgements}

We thank Lixin Zheng, Koneti Rao, and Jack Bleesing for helpful discussions, as well as Andy Snow for critically reading this manuscript. This review is dedicated to the memory of the late Stephen Straus, who led the NIH ALPS group to many new discoveries. This work was supported by the Intramural Research Program of the National Institute of Allergy and Infectious Diseases, NIAID.

This work was supported by the Intramural Research Program of the NIH, National Institute of Allergy and Infectious Diseases

\section{References}

1. Su, HC.; de Oliveira, JB.; Lenardo, MJ. 14. Programmed cell death in lymphocytes. In: Rich, RR.; Shearer, WT.; Fleischer, TA.; Schroeder, HW.; Weyand, CM.; Frew, AJ., editors. Clinical Immunology. Vol. 3. Mosby: 2008. in press

2. Kroemer G, El-Deiry WS, Golstein P, et al. Classification of cell death: recommendations of the Nomenclature Committee on Cell Death. Cell Death Differ Nov;2005 12 (Suppl 2):1463-1467. [PubMed: 16247491]

3. Lenardo M, Chan KM, Hornung F, et al. Mature T lymphocyte apoptosis--immune regulation in a dynamic and unpredictable antigenic environment. Annu Rev Immunol 1999;17:221-253. [PubMed: 10358758]

4. Bleesing JJH. Autoimmune lymphoproliferative syndrome a genetic disorder of abnormal lymphocyte apoptosis. Immunol Allergy Clin N Am 2002;22:339-355.

5. Janka GE. Hemophagocytic syndromes. Blood Rev. Jun 20;2007

6. Bidere N, Su HC, Lenardo MJ. Genetic disorders of programmed cell death in the immune system. Annu Rev Immunol 2006;24:321-352. [PubMed: 16551252]

7. Nichols KE, Ma CS, Cannons JL, Schwartzberg PL, Tangye SG. Molecular and cellular pathogenesis of X-linked lymphoproliferative disease. Immunol Rev Feb;2005 203:180-199. [PubMed: 15661030]

8. Sneller MC, Dale JK, Straus SE. Autoimmune lymphoproliferative syndrome. Curr Opin Rheumatol 2003;15(4):417-421. [PubMed: 12819469]

9. Avila NA, Dwyer AJ, Dale JK, et al. Autoimmune lymphoproliferative syndrome: a syndrome associated with inherited genetic defects that impair lymphocytic apoptosis--CT and US features. Radiology Jul;1999 212(1):257-263. [PubMed: 10405750]

10. Sneller MC, Straus SE, Jaffe ES, et al. A novel lymphoproliferative/autoimmune syndrome resembling murine lpr/gld disease. J Clin Invest 1992;90(2):334-341. [PubMed: 1386609]

11. Sneller MC, Wang J, Dale JK, et al. Clinical, immunologic, and genetic features of an autoimmune lymphoproliferative syndrome associated with abnormal lymphocyte apoptosis. Blood 1997;89(4): 1341-1348. [PubMed: 9028957]

12. Bleesing JJ, Brown MR, Straus SE, et al. Immunophenotypic profiles in families with autoimmune lymphoproliferative syndrome. Blood Oct 15;2001 98(8):2466-2473. [PubMed: 11588044]

13. Rieux-Laucat F, Le Deist F, Hivroz C, et al. Mutations in Fas associated with human lymphoproliferative syndrome and autoimmunity. Science Jun 2;1995 268(5215):1347-1349. [PubMed: 7539157] 
14. Aspinall AI, Pinto A, Auer IA, et al. Identification of new Fas mutations in a patient with autoimmune lymphoproliferative syndrome (ALPS) and eosinophilia. Blood Cells Mol Dis Jun-Aug;1999 25(34):227-238. [PubMed: 10575548]

15. Rao VK, Straus SE. Causes and consequences of the autoimmune lymphoproliferative syndrome. Hematology 2006;11(1):15-23. [PubMed: 16522544]

16. Carter LB, Procter JL, Dale JK, Straus SE, Cantilena CC. Description of serologic features in autoimmune lymphoproliferative syndrome. Transfusion Aug;2000 40(8):943-948. [PubMed: 10960521]

17. Kwon SW, Procter J, Dale JK, Straus SE, Stroncek DF. Neutrophil and platelet antibodies in autoimmune lymphoproliferative syndrome. Vox Sang Nov;2003 85(4):307-312. [PubMed: 14633257]

18. Stroncek DF, Carter LB, Procter JL, Dale JK, Straus SE. RBC autoantibodies in autoimmune lymphoproliferative syndrome. Transfusion Jan;2001 41(1):18-23. [PubMed: 11161240]

19. Fang BS, Sneller MC, Straus SE, Frenkel L, Dale JK, Rick ME. Report of a factor VIII inhibitor in a patient with autoimmune lymphoproliferative syndrome. Am J Hematol Jul;2000 64(3):214-217. [PubMed: 10861820]

20. Wong CS, Arkwright PD, Rieux-Laucat F, Cant AJ, Stevens RF, Judge MR. Childhood linear IgA disease in association with autoimmune lymphoproliferative syndrome. Br J Dermatol Mar;2004 150 (3):578-580. [PubMed: 15030346]

21. Nose M, Nishihara M, Kamogawa J, Terada M, Nakatsuru S. Genetic basis of autoimmune disease in MRL/lpr mice: dissection of the complex pathological manifestations and their susceptibility loci. Rev Immunogenet 2000;2(1):154-164. [PubMed: 11324688]

22. Maric I, Pittaluga S, Dale JK, et al. Histologic features of sinus histiocytosis with massive lymphadenopathy in patients with autoimmune lymphoproliferative syndrome. Am J Surg Pathol 2005;29(7):903-911. [PubMed: 15958855]

23. Teachey DT, Manno CS, Axsom KM, et al. Unmasking Evans syndrome: T-cell phenotype and apoptotic response reveal autoimmune lymphoproliferative syndrome (ALPS). Blood 2005;105(6): 2443-2448. [PubMed: 15542578]Epub 2004 Nov 2412

24. Bazemore AW, Smucker DR. Lymphadenopathy and malignancy. Am Fam Physician Dec;2002 66 (11):2103-2110. [PubMed: 12484692]

25. Brown JR, Skarin AT. Clinical mimics of lymphoma. Oncologist 2004;9(4):406-416. [PubMed: 15266094]

26. Dianzani U, Bragardo M, DiFranco D, et al. Deficiency of the Fas apoptosis pathway without Fas gene mutations in pediatric patients with autoimmunity/lymphoproliferation. Blood Apr 15;1997 89 (8):2871-2879. [PubMed: 9108407]

27. Campagnoli MF, Garbarini L, Quarello P, et al. The broad spectrum of autoimmune lymphoproliferative disease: molecular bases, clinical features and long-term follow-up in 31 patients. Haematologica Apr;2006 91(4):538-541. [PubMed: 16537120]

28. Infante AJ, Britton HA, DeNapoli T, et al. The clinical spectrum in a large kindred with autoimmune lymphoproliferative syndrome caused by a Fas mutation that impairs lymphocyte apoptosis. J Pediatr Nov;1998 133(5):629-633. [PubMed: 9821419]

29. Rieux-Laucat F, Blachere S, Danielan S, et al. Lymphoproliferative syndrome with autoimmunity: A possible genetic basis for dominant expression of the clinical manifestations. Blood Oct;1999 94 (8):2575-2582. [PubMed: 10515860]

30. Bleesing JJ, Brown MR, Novicio C, et al. A composite picture of TcR alpha/beta(+) CD4(-)CD8(-) T Cells (alpha/beta-DNTCs) in humans with autoimmune lymphoproliferative syndrome. Clin Immunol Jul;2002 104(1):21-30. [PubMed: 12139944]

31. Bleesing JJ, Brown MR, Dale JK, et al. TcR-alpha/beta(+) CD4(-)CD8(-) T cells in humans with the autoimmune lymphoproliferative syndrome express a novel CD45 isoform that is analogous to murine B220 and represents a marker of altered O-glycan biosynthesis. Clin Immunol Sep;2001 100 (3):314-324. [PubMed: 11513545]

32. Wang J, Zheng L, Lobito A, et al. Inherited human Caspase 10 mutations underlie defective lymphocyte and dendritic cell apoptosis in autoimmune lymphoproliferative syndrome type II. Cell Jul 9;1999 98(1):47-58. [PubMed: 10412980] 
33. Hao Z, Hampel B, Yagita H, Rajewsky K. T cell-specific ablation of Fas leads to Fas ligand-mediated lymphocyte depletion and inflammatory pulmonary fibrosis. J Exp Med 2004;199(10):1355-1365. [PubMed: 15148335]

34. Stranges PB, Watson J, Cooper CJ, et al. Elimination of antigen-presenting cells and autoreactive T cells by Fas contributes to prevention of autoimmunity. Immunity May;2007 26(5):629-641. [PubMed: 17509906]

35. Broker BM, Kraft MS, Klauenberg U, et al. Activation induces apoptosis in Herpesvirus saimiritransformed T cells independent of CD95 (Fas, APO-1). Eur J Immunol Nov;1997 27(11):27742780. [PubMed: 9394798]

36. Fisher GH, Rosenberg FJ, Straus SE, et al. Dominant interfering Fas gene mutations impair apoptosis in a human autoimmune lymphoproliferative syndrome. Cell 1995;81(6):935-946. [PubMed: 7540117]

37. Wu J, Wilson J, He J, Xiang L, Schur PH, Mountz JD. Fas ligand mutation in a patient with systemic lupus erythematosus and lymphoproliferative disease. J Clin Invest Sep 1;1996 98(5):1107-1113. [PubMed: 8787672]

38. Del-Rey M, Ruiz-Contreras J, Bosque A, et al. A homozygous Fas ligand gene mutation in a patient causes a new type of autoimmune lymphoproliferative syndrome. Blood Aug 15;2006 108(4):13061312. [PubMed: 16627752]

39. Bi LL, Pan G, Atkinson TP, et al. Dominant inhibition of Fas ligand-mediated apoptosis due to a heterozygous mutation associated with autoimmune lymphoproliferative syndrome (ALPS) Type Ib. BMC Med Genet 2007;8:41. [PubMed: 17605793]

40. Oliveira JB, Bidere N, Niemela JE, et al. NRAS mutation causes a human autoimmune lymphoproliferative syndrome. Proc Natl Acad Sci U S A May 16;2007 104(21):8953-8958. [PubMed: 17517660]

41. Holzelova E, Vonarbourg C, Stolzenberg MC, et al. Autoimmune lymphoproliferative syndrome with somatic Fas mutations. N Engl J Med 2004;351(14):1409-1418. [PubMed: 15459302]

42. Rossler J, Enders A, Lahr G, et al. Identical phenotype in patients with somatic and germline CD95 mutations requires a new diagnostic approach to autoimmune lymphoproliferative syndrome. $\mathrm{J}$ Pediatr Nov;2005 147(5):691-694. [PubMed: 16291365]

43. Goldman FD, Vibhakar R, Puck JM, et al. Aberrant T-cell antigen receptor-mediated responses in autoimmune lymphoproliferative syndrome. Clin Immunol Jul;2002 104(1):31-39. [PubMed: 12139945]

44. Bettinardi A, Brugnoni D, Quiros-Roldan E, et al. Missense mutations in the Fas gene resulting in autoimmune lymphoproliferative syndrome: a molecular and immunological analysis. Blood Feb 1;1997 89(3):902-909. [PubMed: 9028321]

45. Chen M, Wang YH, Wang Y, et al. Dendritic cell apoptosis in the maintenance of immune tolerance. Science 2006;311(5764):1160-1164. [PubMed: 16497935]

46. Lim MS, Straus SE, Dale JK, et al. Pathological findings in human autoimmune lymphoproliferative syndrome. Am J Pathol Nov;1998 153(5):1541-1550. [PubMed: 9811346]

47. Lopatin U, Yao X, Williams RK, et al. Increases in circulating and lymphoid tissue interleukin-10 in autoimmune lymphoproliferative syndrome are associated with disease expression. Blood May 15;2001 97(10):3161-3170. [PubMed: 11342444]

48. Ohga S, Nomura A, Takahata Y, et al. Dominant expression of interleukin 10 but not interferon gamma in CD4(-)CD8(-)alphabetaT cells of autoimmune lymphoproliferative syndrome. $\mathrm{Br} \mathbf{J}$ Haematol Nov;2002 119(2):535-538. [PubMed: 12406097]

49. Fuss IJ, Strober W, Dale JK, et al. Characteristic T helper $2 \mathrm{~T}$ cell cytokine abnormalities in autoimmune lymphoproliferative syndrome, a syndrome marked by defective apoptosis and humoral autoimmunity. J Immunol Feb 15;1997 158(4):1912-1918. [PubMed: 9029133]

50. Bleesing JJ, Straus SE, Fleisher TA. Autoimmune lymphoproliferative syndrome. A human disorder of abnormal lymphocyte survival. Pediatr Clin North Am Dec;2000 47(6):1291-1310. [PubMed: 11130997]

51. Bleesing JJ. Sorting out the causes of ALPS. J Pediatr Nov;2005 147(5):571-574. [PubMed: 16291343] 
52. Rao VK, Dugan F, Dale JK, et al. Use of mycophenolate mofetil for chronic, refractory immune cytopenias in children with autoimmune lymphoproliferative syndrome. Br J Haematol 2005;129(4): 534-538. [PubMed: 15877736]

53. Kossiva L, Theodoridou M, Mostrou G, et al. Mycophenolate mofetil as an alternate immunosuppressor for autoimmune lymphoproliferative syndrome. J Pediatr Hematol Oncol Dec; 2006 28(12):824-826. [PubMed: 17164652]

54. Le Deist F, Emile JF, Rieux-Laucat F, et al. Clinical, immunological, and pathological consequences of Fas-deficient conditions. Lancet Sep 14;1996 348(9029):719-723. [PubMed: 8806292]

55. Heelan BT, Tormey V, Amlot P, Payne E, Mehta A, Webster AD. Effect of anti-CD20 (rituximab) on resistant thrombocytopenia in autoimmune lymphoproliferative syndrome. Br J Haematol Sep; 2002 118(4):1078-1081. [PubMed: 12199788]

56. Teachey DT, Obzut DA, Axsom K, et al. Rapamycin improves lymphoproliferative disease in murine autoimmune lymphoproliferative syndrome (ALPS). Blood Sep 15;2006 108(6):1965-1971. [PubMed: 16757690]

57. Benkerrou M, Le Deist F, de Villartay JP, et al. Correction of Fas (CD95) deficiency by haploidentical bone marrow transplantation. Eur J Immunol Aug;1997 27(8):2043-2047. [PubMed: 9295043]

58. Kahwash SB, Fung B, Savelli S, Bleesing JJ, Qualman SJ. Autoimmune lymphoproliferative syndrome (ALPS): a case with congenital onset. Pediatr Dev Pathol Jul-Aug;2007 10(4):315-319. [PubMed: 17638424]

59. Sleight BJ, Prasad VS, DeLaat C, et al. Correction of autoimmune lymphoproliferative syndrome by bone marrow transplantation. Bone Marrow Transplant Aug;1998 22(4):375-380. [PubMed: 9722073]

60. Rao VK, Dowdell KC, Dale JK, et al. Pyrimethamine treatment does not ameliorate lymphoproliferation or autoimmune disease in MRL/lpr-/- mice or in patients with autoimmune lymphoproliferative syndrome. Am J Hematol. Aug 2;2007

61. Straus SE, Jaffe ES, Puck JM, et al. The development of lymphomas in families with autoimmune lymphoproliferative syndrome with germline Fas mutations and defective lymphocyte apoptosis. Blood Jul 1;2001 98(1):194-200. [PubMed: 11418480]

62. Legembre P, Barnhart BC, Zheng L, et al. Induction of apoptosis and activation of NF-kappaB by CD95 require different signalling thresholds. EMBO Rep 2004;5(11):1084-1089. [PubMed: 15514680]

63. Rao VK, Carrasquillo JA, Dale JK, et al. Fluorodeoxyglucose positron emission tomography (FDGPET) for monitoring lymphadenopathy in the autoimmune lymphoproliferative syndrome (ALPS). Am J Hematol 2006;81(2):81-85. [PubMed: 16432855]

64. Vaishnaw AK, Orlinick JR, Chu JL, Krammer PH, Chao MV, Elkon KB. The molecular basis for apoptotic defects in patients with CD95 (Fas/Apo-1) mutations. J Clin Invest Feb;1999 103(3):355363. [PubMed: 9927496]

65. Martin DA, Zheng L, Siegel RM, et al. Defective CD95/APO-1/Fas signal complex formation in the human autoimmune lymphoproliferative syndrome, type Ia. Proc Natl Acad Sci U S A Apr 13;1999 96(8):4552-4557. [PubMed: 10200300]

66. Siegel RM, Frederiksen JK, Zacharias DA, et al. Fas preassociation required for apoptosis signaling and dominant inhibition by pathogenic mutations. Science Jun 30;2000 288(5475):2354-2357. [PubMed: 10875918]

67. Siegel RM, Muppidi JR, Sarker M, et al. SPOTS: signaling protein oligomeric transduction structures are early mediators of death receptor-induced apoptosis at the plasma membrane. J Cell Biol Nov 22;2004 167(4):735-744. [PubMed: 15557123]

68. Roesler J, Izquierdo JM, Ryser M, et al. Haploinsufficiency rather than the effect of an excessive production of soluble CD95 (CD95 Delta\}TM) is the basis for ALPS Ia in a family with duplicated 3' splice site AG in CD95 intron 5 on one allele. Blood Sep 1;2005 106(5):1652-1659. [PubMed: 15870181]

69. Cerutti E, Campagnoli MF, Ferretti M, et al. Co-inherited mutations of Fas and caspase-10 in development of the autoimmune lymphoproliferative syndrome. BMC Immunol Nov;2007 8(1):28. [PubMed: 17999750] 
70. Jackson CE, Fischer RE, Hsu AP, et al. Autoimmune lymphoproliferative syndrome with defective Fas: genotype influences penetrance. Am J Hum Genet 1999;64(4):1002-1014. [PubMed: 10090885]

71. van der Burg M, de Groot R, Comans-Bitter WM, et al. Autoimmune lymphoproliferative syndrome (ALPS) in a child from consanguineous parents: a dominant or recessive disease? Pediatr Res Mar; 2000 47(3):336-343. [PubMed: 10709732]

72. Kasahara Y, Wada T, Niida Y, et al. Novel Fas (CD95/APO-1) mutations in infants with a lymphoproliferative disorder. Int Immunol Feb;1998 10(2):195-202. [PubMed: 9533447]

73. Zhu S, Hsu AP, Vacek MM, et al. Genetic alterations in caspase-10 may be causative or protective in autoimmune lymphoproliferative syndrome. Hum Genet 2006;119(3):284-294. [PubMed: 16446975]Epub 2006 Jan 2031

74. Gronbaek K, Dalby T, Zeuthen J, Ralfkiaer E, Guidberg P. The V410I (G1228A) variant of the caspase-10 gene is a common polymorphism of the Danish population. Blood Mar 15;2000 95(6): 2184-2185. [PubMed: 10755819]

75. Peters AM, Kohfink B, Martin H, et al. Defective apoptosis due to a point mutation in the death domain of CD95 associated with autoimmune lymphoproliferative syndrome, T-cell lymphoma, and Hodgkin's disease. Exp Hematol May;1999 27(5):868-874. [PubMed: 10340403]

76. Mateo V, Menager M, de Saint-Basile G, et al. Perforin-dependent apoptosis functionally compensates Fas-deficiency in Activation-induced cell-death of human T-lymphocytes. Blood. Aug; 2007

77. Clementi R, Chiocchetti A, Cappellano G, et al. Variations of the perforin gene in patients with autoimmunity/lymphoproliferation and defective fas function. Blood. May 23;2006

78. Clementi R, Dagna L, Dianzani U, et al. Inherited perforin and Fas mutations in a patient with autoimmune lymphoproliferative syndrome and lymphoma. N Engl J Med Sep 30;2004 351(14): 1419-1424. [PubMed: 15459303]

79. Rieux-Laucat F, Le Deist F, De Saint Basile G. Autoimmune lymphoproliferative syndrome and perforin. N Engl J Med Jan 20;2005 352(3):306-307. [PubMed: 15659737]author reply 306-307

80. Lobito AA, Kimberley FC, Muppidi JR, et al. Abnormal disulfide-linked oligomerization results in ER retention and altered signaling by TNFR1 mutants in TNFR1-associated periodic fever syndrome (TRAPS). Blood Aug 15;2006 108(4):1320-1327. [PubMed: 16684962]

81. Chiocchetti A, Indelicato M, Bensi T, et al. High levels of osteopontin associated with polymorphisms in its gene are a risk factor for development of autoimmunity/lymphoproliferation. Blood Feb 15;2004 103(4):1376-1382. [PubMed: 14592838]

82. Chun HJ, Zheng L, Ahmad M, et al. Pleiotropic lymphocyte activation defects due to caspase-8 mutation cause human immunodeficiency. Nature September 26;2002 419:395-399. [PubMed: 12353035]

83. Su H, Bidere N, Zheng L, et al. Requirement for caspase- 8 in NF-kappaB activation by antigen receptor. Science 2005;307(5714):1465-1468. [PubMed: 15746428]

84. Varfolomeev EE, Schuchmann M, Luria V, et al. Targeted disruption of the mouse Caspase 8 gene ablates cell death induction by the TNF receptors, Fas/Apo1, and DR3 and is lethal prenatally. Immunity Aug;1998 9(2):267-276. [PubMed: 9729047]

85. Salmena L, Lemmers B, Hakem A, et al. Essential role for caspase 8 in T-cell homeostasis and Tcell-mediated immunity. Genes Dev 2003;17(7):883-895. [PubMed: 12654726]Epub 2003 Mar 2021

86. Lemmers B, Salmena L, Bidere N, et al. Essential role for caspase-8 in Toll-like receptors and NFkappaB signaling. J Biol Chem Mar 9;2007 282(10):7416-7423. [PubMed: 17213198]

87. Salmena L, Hakem R. Caspase- 8 deficiency in T cells leads to a lethal lymphoinfiltrative immune disorder. J Exp Med Sep 19;2005 202(6):727-732. [PubMed: 16157684]

88. Salzer U, Grimbacher B. Common variable immunodeficiency: The power of co-stimulation. Semin Immunol Dec;2006 18(6):337-346. [PubMed: 17023177]

89. Ochs HD, Thrasher AJ. The Wiskott-Aldrich syndrome. J Allergy Clin Immunol Apr;2006 117(4): 725-738. [PubMed: 16630926]quiz 739

90. Orange JS, Jain A, Ballas ZK, Schneider LC, Geha RS, Bonilla FA. The presentation and natural history of immunodeficiency caused by nuclear factor kappaB essential modulator mutation. $\mathrm{J}$ Allergy Clin Immunol Apr;2004 113(4):725-733. [PubMed: 15100680] 
91. Marsh RA, Snow AL, Roehrs P, et al. X-linked lymphoproliferative disease (XLP) is a disorder of abnormal lymphocyte function and homeostasis independent of EBV infection. Submitted

92. Chen G, Tai AK, Lin M, Chang F, Terhorst C, Huber BT. Increased proliferation of CD8(+) T cells in SAP-deficient mice is associated with impaired activation-induced cell death. Eur J Immunol Feb 1;2007 37(3):663-674. [PubMed: 17266174] 


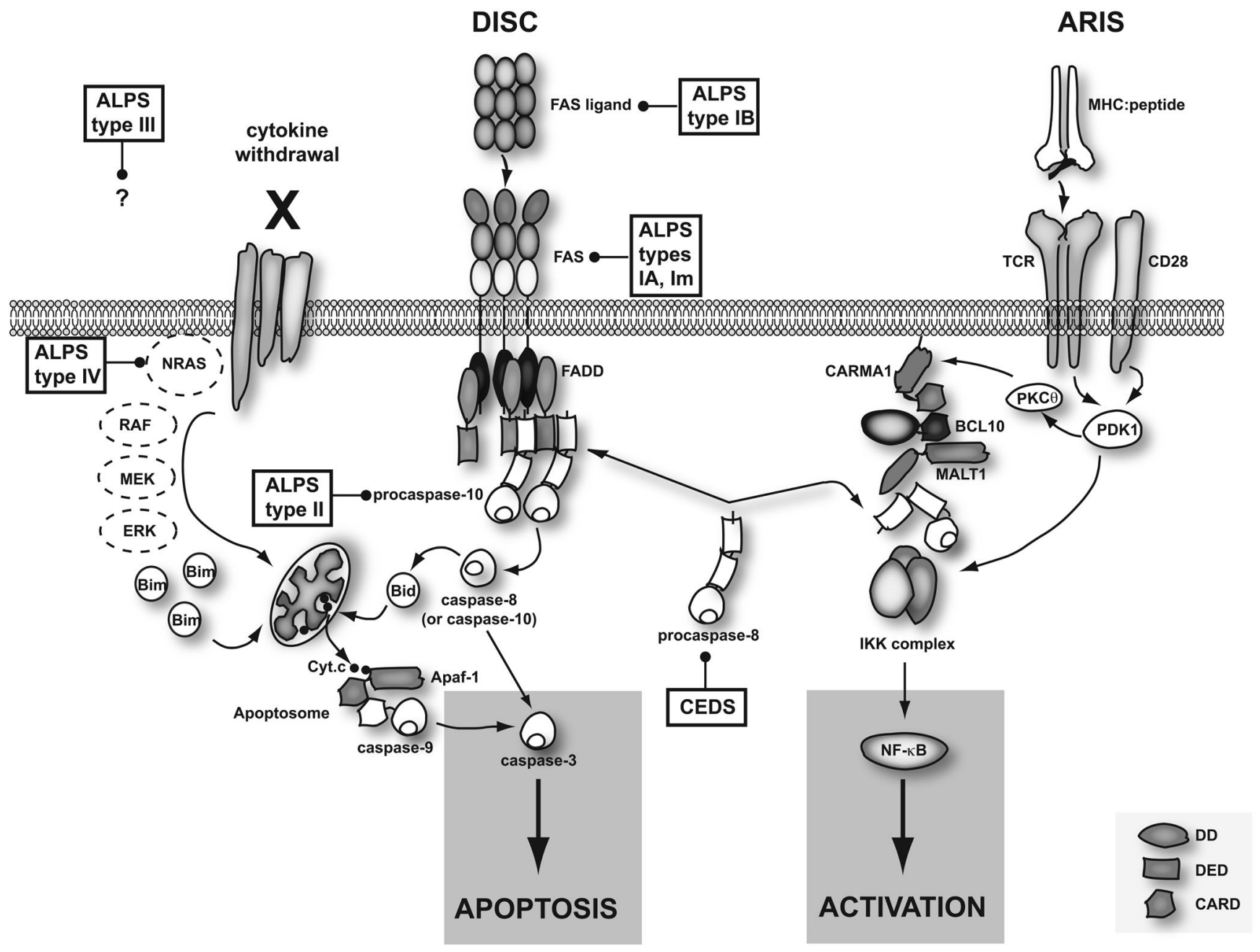

Figure 1.

ALPS and CEDS illustrate signaling pathways for lymphocyte apoptosis and activation. In the death-inducing signaling complex (DISC) (center of figure), death receptor stimulation, here typified by trimerized Fas, leads to recruitment of FADD adapter molecules, which in turn recruits the initiator caspases-8 (and/or caspase-10). Oligomerization causes caspase-8/-10 activation and cleavage of downstream effector caspases. Caspase- 8 cleavage of BID, a proapoptotic member of the BCL-2 family, feeds into a mitochondrial amplification loop in which apoptosome formation oligomerizes and activates caspase-9. During cytokine-withdrawalinduced apoptosis (left side of figure), interruption of IL-2-stimulated signaling leads to decreased activation of the RAF/MEK/ERK pathway. This causes increased expression of BIM, another pro-apoptotic member of the BCL-2 family, which in turn triggers mitochondrial depolarization. Released cytochrome $\mathrm{c}$ from depolarized mitochondria assembles with Apaf-1 and caspase- 9 to form the apoptosome. Both the death receptor- and cytokine withdrawalinduced apoptosis pathways converge following activation of initiator caspases: these cleave and activate downstream effector caspases, which in turn cleave intracellular substrates, causing cell death. In the activation receptor induced signalosome (ARIS) (right side of figure), immunoreceptor stimulation leads to caspase-8-dependent recruitment of the CARMA1BCL10-MALT1 (CBM) complex with the IKK complex. Caspase-8 linker and enzymatic function are required for optimal downstream NF- $\mathrm{kB}$ induction for lymphocyte activation. Mutations in the indicated signaling components that cause ALPS or CEDS are indicated. 
Death domains (DD), death effector domains (DED), and caspase recruitment domains (CARD) in relevant proteins containing these structures are as indicated. 
ALPS classification by gene mutation

Table 1

ALPS classification by gene mutation

\begin{tabular}{|c|c|c|c|}
\hline \multirow[b]{2}{*}{ ALPS type } & \multirow[b]{2}{*}{ Gene mutation } & \multicolumn{2}{|c|}{ Results of apoptosis testing } \\
\hline & & Fas-induced apoptosis & IL-2 withdrawal-induced apoptosis \\
\hline IA & TNFSF6 (FAS) & defective & normal \\
\hline IB & TNFSRF6 (FASLG) & $\begin{array}{l}\text { defective when patient cells used as stimulus to } \\
\text { kill Fas-expressing cell line }\end{array}$ & normal \\
\hline $\mathrm{I}_{\mathrm{m}}$ & somatic TNFSF6 (FAS) & normal & normal \\
\hline II & CASP10 & defective & normal \\
\hline IV & NRAS & normal & defective \\
\hline III & unknown & \multicolumn{2}{|l|}{ defective to at least one stimulus } \\
\hline
\end{tabular}

